\title{
Living with Light: an ethnographic study of older people's use and experience of lighting at home
}

\author{
S.L.Nielsen ${ }^{1}$ and M.F.Mullins ${ }^{1}$ \\ ${ }^{1}$ Aalborg University, Copenhagen, Denmark \\ stm@create.aau.dk mullins@create.aau.dk
}

\begin{abstract}
This ethnographic study aims to better understand the culture, values and everyday life of elderly people in view of their use of lighting in their homes. By addressing different types of lighting and exploring when, how and why older people used various lighting sources in their homes, the study seeks new insight into and an in-depth understanding of how older people experience light in their everyday lives. The paper introduces the results of a qualitative analysis of the collected data and presents some observations on the values and meanings the elderly attach to light and lighting in their daily lives. It is argued that these patterns are largely shaped by the elderly's bodily, social and cultural experiences and contexts, so a framework encompassing four major themes is proposed: 1. Traditions, Economy and Environment; 2. Quality of Life and Independence; 3. Health; and 4. Security and Safety.
\end{abstract}

\section{Introduction}

Aiming to develop better lighting-control tools for older people, the study seeks to better understand the culture, values and everyday life of older people living with lighting in their own homes. Through an examination of how many and what types of lighting sources older people have in their homes, when, how and why such sources are used, as well as how the elderly control and operate lighting, the project has sought new insight into and an in-depth understanding of how older people use and experience light in their everyday lives. The overall data and analysis of the report provide an indication of trends in lighting practices and the values of elderly people at home.

The study is part of a project, which seeks to address and remedy the challenges older people experience with the control of daylight and artificial light, particularly concerning programmable LED, circadian rhythm systems and other new technologies that are becoming widespread in Denmark. By asking the question of "How the elderly experience lighting in their everyday experience", the study employs ethnographic methods that, through a qualitative phenomenological approach, explore older people's experiences with lighting and their use of light in everyday life.

Two anthropologists facilitated three focus groups with 18 older citizens aged 61-80 years in cooperation with a local municipality. The citizens met at the Town Hall for an in-depth reflection and discussion of their everyday lives with light and lighting, focusing on their needs, wishes and ideas for the control and operation of light. 
Prior to the focus group meeting, the participants had received three home assignments (or tasks), which they had been requested to prepare before the meeting. This form of data collection is inspired by design-anthropological methods (Gaver, Dunne, \& Pacenti, 1999), which seek to open up participants' reflections on their everyday practices and experiences, in this case, with lighting at home.

The home assignments consisted of three tasks:

1. Where I live and where I have light

2. My day-today experience with light

3. Reflections about use of lighting in my home

The first assignment served as a form of mapping of the elderly's homes, where the elderly outlined a plan of their housing and its various forms, while specifying the place and number of light sources. The second task included a description of the space, activities and light sources the elderly used over 24 hours. Inspired by anthropological data collection methods on practice (Lange, 1988), the first and second home assignment thus worked together as a form of a guided tour for the participants' everyday life with light at home. The third task required the participants to complete a reflection sheet by answering the following questions:

1) In my everyday life, light means to me...?

2) Without light in my everyday life, I would...?

3) Light has a special significance for me when I...?

All 18 participants in the focus groups completed their three home assignments. The statistical and qualitative data obtained from the home assignments are based on 16 valid answers received from 17 older people and the data collected from the focus groups (18 participants).

Based on the home assignments, the focus groups were then used as an exploratory ethnographic method of translating, discussing and uncovering different values and practices that older people live with, and their connections to lighting at home. In particular, the study was interested in the meanings the target group attributed to lighting, their understanding of technical differences between LEDs and other light sources, as well as the link between light, aging and circadian rhythms (Sen et al., 2017).

Two anthropologists moderated the discussion in the group based on a questionnaire that allowed attitudes and views associated with home illumination to be debated; everyday experiences with light to be described and compared; and needs, wishes and ideas for the control of light to be discussed (Lange, 1988).

The first half of the focus group interview consisted of examining differences and similarities in the participants' answers to the home assignments. Here, the participants' values, habits, rhythms, routes and routines were associated with light in everyday life.

The second part of the focus group consisted in uncovering the current possibilities and limitations of the elderly for controlling the light in their homes, thereby exploring their needs, ideas, and wishes for controlling the light. A supplier ${ }^{1}$ of lighting concepts and control systems for the healthcare sector had lent three different types of light controls that the elderly could study and discuss. Similar to the first part of the focus group, the participants' reflections and perspectives were facilitated by two anthropologists, with the help of a questionnaire.

The debates in the focus groups, together with the participants' answers to the home assignments, provide insights into the lives of the elderly with lighting at home. The processing and description of data has been structured through the distinction between light as a materiality, as a practice and finally as a value. Taken together, these distinctions form a picture of the lives of the elderly with light at home.

\footnotetext{
${ }^{1}$ Chromaviso A/S
} 


\section{Light as materiality in the home}

\subsection{Number and location of light sources in the home}

The primary rooms of the elderly's homes include the entrance hall, living room, kitchen, bathroom, bedroom and work space. These rooms thus appear on all floor plans presented by the elderly as part of the first assignment.

The secondary rooms of the elderly's homes include the dining room, utility room / family room, guest room, hallway, staircase, terrace and / or orangery. Six of the elderly live in homes that include these rooms, which can point to the elderly's housing situation - the size and partly type of housing (an apartment or a house).

The data show that 17 older people have 543 light sources in their homes - an average of 32 light sources per home. Generally, data shows that the biggest number of light sources used by the older people are located in the living room. In total, 17 older people have 115 light sources in their living rooms, an average of seven light sources per living room, ranging from max. 14 to minimum two sources. This observation largely depends on the fact that the living room is typically the largest and most multifunctional home space. In other words, the living room serves for more elderly people as a room that provides a framework for several types of practices that consequently require different types of lighting.

The kitchen is a place, where the second biggest amount of light sources is concentrated, with 84 light sources combined. The average of five light sources per kitchen, according to the data collected from the focus group interviews, is often due to the location of skylights and spots in the ceiling and under the kitchen cabinets, respectively. In the bedroom, the elderly have, on average, three light sources located, primarily in the form of one skylight and several reading lamps.

Halls and bathrooms are the rooms generally contain relatively few light sources. In addition, there is a tendency for secondary rooms in the homes of the elderly (e.g. corridors, stairs, guest rooms, dining rooms and utility rooms) to accommodate between 11 to 25 light sources in total.

\subsection{The nature of home light sources}

As part of the first home assignment 1, the elderly were asked to indicate the nature of their light sources in their homes, subjectively classifying them as 'sharp', 'less sharp' or 'weak'. Overall, the home assignments showed that the elderly primarily see their homes as mostly using sharp light sources (227 light sources), followed by less sharp light sources (196 light sources) and less sharp light sources (111 light sources).

According to the elderly's feedback from the focus group, when it comes to the nature of the home light sources, a distinction is made between "functional lights" and "cozy lights", or "mood lights". The elderly thus expressed their preferences in terms of how they placed and used different types of light sources to support them in different activities and moods at home.

The distribution of different types of home light sources by the elderly shows a tendency for the placement of predominantly sharp light sources in the rooms with a specific fixed functionality, such as cooking in the kitchen and working in the office space. The living room and bedroom, on the other hand, are characterized by a preponderance of medium-glare sources and an almost even distribution between sharp and soft lighting, which defines this space as more fluid, where more varied activities can take place.

In relation to the nature of light sources at home, a tendency for a specific light culture among the elderly was detected, with the majority of the elderly having light placed on / in shelves and onto paintings, although, according to several participants, this is a sign of "belonging to the generation that came before 
us". In addition, participants mentioned the challenge in finding compatible light sources for the $\mathrm{PH}^{2}$ lamps that many have in their homes. This "PH problem", as one could call the challenge, arises, as one elderly participant explained, in the encounter between the diffusion of light in the lamp design, the brightness of the light source and the heat released by the luminaire. The new types of light sources, such as LEDs, are simply unable to deliver a sufficiently high quality of light through the PH lamp luminaire and often overheat. In addition, the participants also explained how the quality of light in many of the new light sources did not have sufficient color reproduction allowing one to distinguish between various colors and patterns when, for example, knitting or sewing. The PH problem was generally addressed by the older men during the focus groups, and the female participants expressed their opinion that the PH lamp light is of poor quality.

The attitudes to sharp and cold light vs. soft and warm light were also reflected in the participants' answers to the third assignment. For example, one participants provided the following response to the question: 'Light had a special significance for me when I ...?'"Modern cars with sharp LED white / bluish light dazzle me on the dark road. A Christmas tree with warm electric lighting makes me happy. If it has a bright white / bluish light, it does something negative to me ..." Another participant mentioned how her neighbour had once pointed out how much glare the light in the kitchen gave. Since then she was therefore extra careful to use this light. Another participant, in contrast, said that he appreciated the "warm blue light", which may be due to the fact that he earlier lived in Asia, so he had a higher degree of exposure to this type of lighting.

Thus, the specific patterns and values of different forms of light among the elderly were observed, which can be understood, inter alia, in the context of gender, social and cultural background.

\section{Light as a practice in the home}

The qualitative data regarding the number and types of light sources the elderly have at home, hides a more qualitative, phenomenological insight into the ways the elderly use light sources in practice and how they inhabit their homes.

In order to address and understand the role and importance of the home lighting for the elderly, and based on this input create lighting controls suitable for the elderly, it is essential to address the home as a cultural phenomenon and a context for their everyday life with light.

From an anthropological, phenomenological perspective, home is not something you have, but something you do. In other words, a home "happens" through human beings dwelling in physical frameworks, as a relational process between humans and materiality, where people through their own rhythms, routes and routines allocate physical frameworks and life in themselves (Ingold, 1995).

In this understanding of the concept of home, lighting and the control of light in the home of the elderly thereby not only expresses a limited materiality but more importantly, comprise elements within a larger whole with which the elderly can inhabit their physical surroundings. Ultimately, lighting assists people to shape their experience and meaning of home.

With this view in mind, data from the homework of the elderly and the statements in the focus group were analyzed through a study of both individual and ongoing rhythms, routes and routines, which in practice make up the lives of the elderly with light at home.

\footnotetext{
${ }^{2}$ Poul Henningsen lamp: PH lamps were originally designed in the 1920 s, but are still very common among all age groups in Danish homes today.
} 


\subsection{Rhythms}

When reviewing the participants' homework, we observed a tendency for a lack of active awareness about which, when and how many light sources are used. For example, where the elderly listed the light souces they used in the second homework assignment, generally these observations were made when the sources were turned on, rather than observing for how long they remained lit.

From another perspective, the feedback from the second homework assignment demonstrates that in practice the elderly use the light sources in the home as a form of background for living their lives, where lighting has merged with the background of their immediate awareness. In other words, the interaction with light seems to be based on what could be called a "course of behavior", where the use of light has become a matter of course and invisible to the elderly through repetitive rhythms and habits - just as people's use of materiality in everyday life generally seems to be (Latour, 1992; Miller, 2010).

\subsection{Routes}

To further understand the use of home light sources by the elderly, we carried out a mapping of the rooms that the participants used at various times of the day. This mapping indicated that the bedroom, bathroom, kitchen and living room are primarily used in the morning, whereas the living room, kitchen and office are used in the afternoon. The participants mainly (and almost only) use the kitchen and the living room in relation to cooking and dining, and from $7 \mathrm{pm}$ until bedtime the primary (and most importantly) used room is the living room. The rooms used late in the day are the living room, bathroom and bedroom, the latter from $10 \mathrm{pm}$ and later.

\subsection{Routines}

In addition to the continuous rhythms and routes that have appeared in the data relating to the elderly living with light, some participants in the focus group reported on their individual "home routines" in relation to their use of light. In the words of a participant: "When I get home, I always do my route and light candles ... I have my very particular rhythm". This special routine consisted of turning on the table lamps and lamps around the living room, as well as turning on the light in the workroom. Overall, the data obtained from both home tasks and statements from the focus group showed that the elderly illuminate the rooms that they use actively both as a way to illuminate the space used and as a way to create an overview of their surroundings. One participant explained it -as follows:"It's a bit like when I turn on the lights in the corners of the living room .. you just need to create a place. it gives a sense of security". In addition, some participants mentioned how the "lighting the lights" always happens when guests are visiting.

These rhythms and routines are significant in forming the concept of 'being at home' and are important to include in the design of home lighting and lighting control.

\section{Light as a home value}

In the analysis of the assignments and debate in the focus group, several key themes and values emerged. These are the themes which seem to link the elderly's experience, understanding and use of light at home.

\subsection{Tradition, environment and economy}

The majority of the participating elderly were surprisingly well informed and well versed about the recent developments of light sources and LED technology. In this context, it should be pointed out that the 
participating elderly as a group should not be considered uncritically representative of the older Danish population as a whole, as all of the participants had joined the focus groups on the basis of their interest in lighting control at home.

Despite their knowledge of and use of new LED technology at home, the majority of the participants explained how they had experienced challenges with the recent years' technological transition from incandescent bulbs to LED bulbs. The elderly were generally fond of the traditional incandescent bulb that they had been used to and which "give such a nice warm light. The shift to a newer lighting technology was thus not compatible with values such as 'recognisability', 'usability' and 'tradition'.

In addition, many of the participants said that the new light sources could be difficult to integrate in their current lighting at home. This left several of the participants with a variety of lighting solutions at home, which some perceived as an irritating element.

Otherwise, the participants understood that the transition to LED bulb had led to greater economic savings, better environmental practices and a reduction in energy consumption. In addition, the participants reported that their participation in the focus group was based on their interest in gaining more knowledge about lighting and more strategies to live a "greener" life and lessen their impact on the environment with their individual light practices.

Thus, values such as 'recognizableness', 'usability', 'tradition', 'economical saving' and 'environment' are significant among the elderly in terms of their experience and use of light sources. In addition, the experience of poor light quality and high purchase prices seem to be among the perceived disadvantages of LED.

\subsection{Life quality and personal control}

The elderly explained how light is a central part of their everyday life, and how it supports their ways of home living. The elderly live with an experience and use of light as an essential element of staging and support of activities and moods at home.

Several older people pointed out how they used different light sources for different activities and moods. Bright light is most often used for the activities that focus on detail, such as cooking, reading and handiwork, whereas soft, reddish, "cozy lighting" is used in the living room in the evening and / or when there are visitors. One participant stated how "all activities go faster where the light complements them" and another expressed how she "... has bright light to work, read, knit and put make-up on; and more muted light to give coziness and security." More generally, others expressed how they would "feel the room uncomfortable and hard to read" and that it "would not work - neither for activities or for pleasure".

About half of the elderly had installed dimmers at home, especially in the living room, which for most people forms a framework for several different activities and serves as a scene for different moods. The distinction between the use of functional, mood lighting, and dimming possibilities in everyday life indicates that the elderly put a value in adjusting the lighting to suit them, the activity or the mood they are in or want to create. Thus, they broadly associate light with an experience of quality of life and wellbeing. Their experience of quality of life also seems to be connected to the opportunity and experience of personal control, and ability to adjust lighting. Flexibility and access to control of light is crucial to the elderly's experience of the quality of life associated with being the master of one's own house and one's own life.

\subsection{Safety and security}

The participants describe collectively how the light allows them to experience a sense of security in their own homes, both in terms of the confidence they experience with lighting at home in repeated bodily rhythms, routes and routines, but also through their ability to sense their surroundings and have a home. 
In other words, the home's well-known practices and physical materiality provide an experience of safety for the elderly.

Additionally, light contributes to safety issues among the elderly in terms of accidents and falls. Proposals from both the focus groups and the home assignments showed how more elderly people had installed bright light at home. "I need to see the steps, so I do not stand on my heads going down the stairs", one participant said. Another participant mentioned how the fact that visual impairment made it difficult for her to navigate at home, with dim lighting.

\subsection{Health and well-being}

The data collected from the focus groups and the home assignments show how home lighting was influenced by the health of the elderly. Several of the elderly expressed that "light and well-being are connected". In particular, the influence of light on daily rhythm was something that emerged, largely linked to the daily rhythm of the natural light (Sen et al., 2017).

From the viewpoint of light as a practice, the opportunity for the elderly to control and adjust lighting through physical practice (see rhythms, routes and routines above) is not only significant in relation to the creation, maintenance and experience of home but is also significant in relation to the creation, maintenance and experience of health and well-being.

\subsection{Summary}

In general, the analysis of the collected data shows how older people use home light sources as a form of supportive background for their lives. To this end, the elderly themselves distinguish between basic, work and mood lighting, which they apply and adjust to their desires for activities and atmospheres, but which they often use simultaneously.

The recorded experiences provide an insight into what values and meanings the elderly attach to light in their daily lives, largely shaped by their bodily, social and cultural experiences and context. These values were grouped in the four categories: Traditions, Economy and Environment; Quality of Life and Independence; Health; and Security and Safety. 


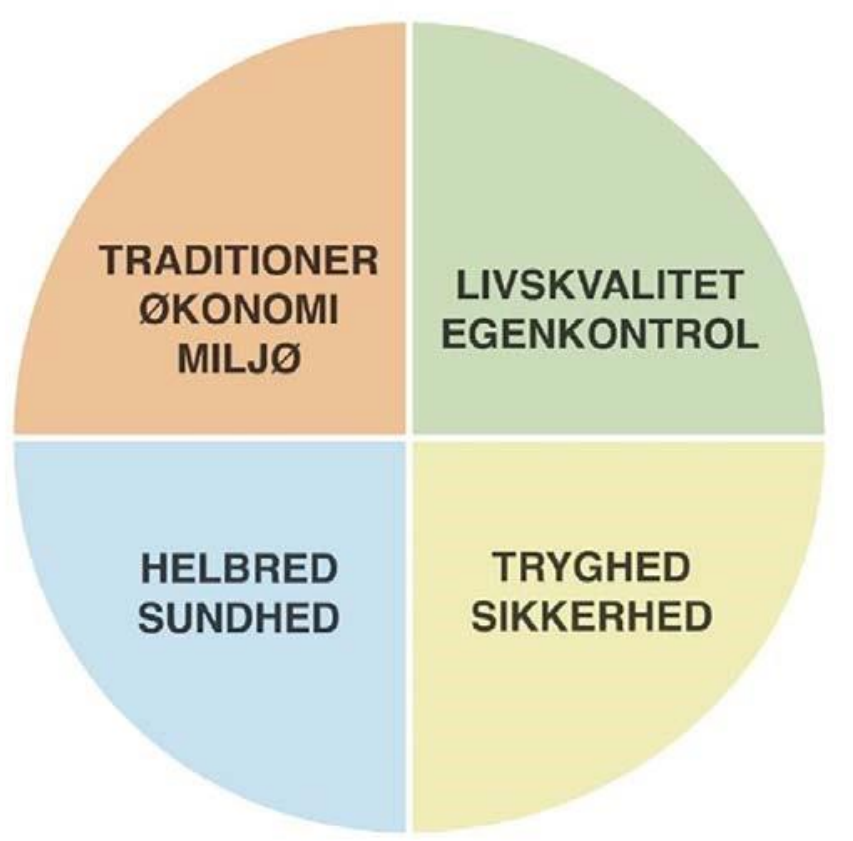

Figure 1: Model of Lighting Values for the Elderly

Experiences of technology, economy and environment were prominent and while several participants saw many benefits to technological development of lighting, the overall attitude was that the change seems to be too fast and does not offer any real useful advantage. In addition, participants experienced new lighting solutions as economically costly compared to what they have been used to; and they looked for information that would enable them to exploit the technologies toward greater care for the environment.

In addition, cognitive and sensory changes that accompany ageing play an important role, which designers need to take into account, in terms of safety and health.

\section{Discussion}

The qualitative data regarding the number and types of light sources the elderly have at home unveils a phenomenological insight into the way how elderly people use light sources in practice, and how light influences the way they inhabit their living space. In order to address and understand the role and importance of lighting for the elderly, and from there, create lighting controls, it is essential to address the home as a cultural phenomenon and context for their living of everyday life with light. From an anthropological, phenomenological perspective, home is not something one has, but something one does. In other words, a home "happens" through people's acquisition of, and dwelling in, physical frameworks - as a relational process between humans and materiality, where people allocate meaning to physical frameworks and life itself through their own rhythms, routes and routines.

The study's observations provide the insights, which are useful for the design and operation of lighting control for the elderly. An understanding of light as materiality, practice and value in the home of the elderly promotes awareness of the fact that lighting control is not a fragmented, isolated element in the everyday life of the elderly, but part of coherent everyday life and practice of home. The development of tools for controlling light thus can ultimately affect this greater context. 
When creating light control technologies, the everyday home lighting practices can be changed, including the rhythms, routes and routines. Thus, lighting control has the potential to influence the practice of home, the experience of being-in-control, safety and security, as well as the sense of health and the overall quality of life among the elderly.

The development of light-controls for the elderly requires a particular ethical awareness and responsibility to protect and keep the user's home practice in mind. Lighting control devices should not be developed merely because technology allows it, but with a sense of sensitivity to and a desire for optimization and support for the reality that it shapes.

\section{Acknowledgements}

The authors wish to express their gratitude to the older citizens in Gladsaxe Municipality, who took the time to get involved in the assignments and the focus group. We are also grateful to Chromaviso for lending the equipment and to Gladsaxe Municipality's engagement and enthusiasm in organizing the focus group and providing the venue. In addition, we are thankful to anthropologist Nanna Hauge Kristensen for her constructive assistance during the study's data collection and processing. The study described in this paper is part of the project called 'Better Light - Better Operation', financed by the Danish Lighting Innovation Network.

\section{References}

Gaver, B., Dunne, T., \& Pacenti, E. (1999). Design: Cultural probes. Interactions, 6(1), 21-29. https://doi.org/10.1145/291224.291235

Ingold, T. (1995). Building, dwelling, living: how animals and people make themselves at home in the world. In Shifting contexts: Transformations in anthropological knowledge (pp. 57-80).

Lange, B. K. (1988). Ethnographic Interview. Occupational Therapy in Mental Health, 8(2), 6180. https://doi.org/10.1300/J004v08n02_05

Latour, B. (1992). Where are the missing masses, sociology of a few mundane artefacts application. In Shaping Technology-Building Society. Studies in Sociotechnical Change (pp. 225259). Cambridge Mass.: MIT Press.

Miller, D. (2010). Theories of Things. In Stuff (pp. 42-78). Cambridge: Polity Press.

Sen, S., Flyvholm, A., Xylakis, E., Nielsen, S. M. L., Hansen, E. K., Mullins, M. F., \& Triantafyllidis, G. (2017). Towards assessing the impact of circadian lighting on the well-being of the elderly from a holistic perspective. In 3rd International Conference on Architecture, Research, Care, Health (pp. 1-20). Polyteknisk Forlag. 